\title{
Estimation and correction for the effect of sound velocity variation on GPS/Acoustic seafloor positioning: An experiment off Hawaii Island
}

\author{
Yukihito Osada $^{1}$, Hiromi Fujimoto ${ }^{2}$, Satoshi Miura ${ }^{2}$, Aaron Sweeney ${ }^{2}$, Toshihiko Kanazawa ${ }^{1}$, Shigeru Nakao ${ }^{1}$ \\ Shin-ichi Sakai ${ }^{1}$, John A. Hildebrand ${ }^{3}$, and C. David Chadwell ${ }^{3}$ \\ ${ }^{1}$ Earthquake Research Institute, University of Tokyo, 1-1-1 Yayoi, Bunkyo-ku, Tokyo 113-0032, Japan \\ ${ }^{2}$ Graduate School of Science, Tohoku University, Aramaki, Aoba-ku, Sendai 980-8578, Japan \\ ${ }^{3}$ Scripps Institution of Oceanography, University of California San Diego La Jolla, CA 92093-0205, USA
}

(Received September 19, 2003; Revised November 3, 2003; Accepted November 5, 2003)

\begin{abstract}
A GPS/Acoustic experiment on the southeastern slope of Hawaii Island presented precise seafloor positioning in the condition of large water depth $(2.5-4.5 \mathrm{~km})$ and large velocity variations. We estimated sound velocity variations from acoustic ranging, and found that temperature variation can well explain the velocity variation. The effect of daily variation in the sound velocity amounted to $+/-0.7 \mathrm{~m}$ on acoustic ranging of $4-7 \mathrm{~km}$ with a fixed velocity structure. CTD data observed about every 3 hours could decrease the range residuals to $+/-0.4 \mathrm{~m}$. These large residuals were fairly well canceled in the positioning of the array center of three acoustic transponders. The estimated precision of the array center positioning was about $3 \mathrm{~cm}$ in latitude and longitude.
\end{abstract}

Key words: GPS/Acoustic, seafloor positioning, acoustic ranging, sound velocity, Hawaii Island.

\section{Introduction}

Recent results of various geodetic observations near seafloor spreading centers suggest episodic deformations on plate boundaries (e.g., Chadwell et al., 2002; Fujimoto et al., 2003). Deformations in subduction zones remain to be clarified, and a combination of sea surface GPS positioning with underwater acoustic positioning (GPS/A positioning) is the most basic approach to the observation. Probably, the experiment will become challenges to observe at deeper area and at the vicinity of coast where tidal sea variations are expected to be large.

Our group from three institutions jointly developed a precision, digital acoustic ranging system for deep seafloor (Fujimoto et al., 2001). Scripps Institution of Oceanography, University of California, started a GPS/A experiment for monitoring crustal deformation on the southeastern slope of Hawaii Island by using the same acoustic system, and deployed 7 precision acoustic transponders (PXPs) as is shown in Fig. 1. The area is on a long submarine slope, and ocean tides may bring about daily variations in sound velocity structures. These features are similar to those of subduction zones. We therefore joined the first cruise of the experiment in 2000 to examine the newly developed acoustic system and the effect of velocity variations. We added one PXP (DJ1) and carried out GPS/A observation by using 3 PXPs: D8, D9, and DJ1 deployed at depths of 2,500-4,500 m. We report here mainly on the effect of sound velocity variations on precise seafloor positioning.

Copy right(C) The Society of Geomagnetism and Earth, Planetary and Space Sciences (SGEPSS); The Seismological Society of Japan; The Volcanological Society of Japan; The Geodetic Society of Japan; The Japanese Society for Planetary Sciences.

\section{GPS and Acoustic Observations}

A system for the GPS/A observation consisted of three components: GPS positioning, acoustic ranging, and data processing. We set 3 GPS antennas on the ship and a reference at Hivalt (Fig. 1) at an altitude of about 1,250 m. Sampling interval was 1 second for the logging both on land and at sea. The baseline length for kinematic GPS positioning was about $50 \mathrm{~km}$.

The surface acoustic unit (SAU) transmits an acoustic signal at a timing of $1 \mathrm{~Hz}$ pulse sent from a GPS receiver. The PXPs on the seafloor return the signal with a fixed delay time of $26.625 \mathrm{~ms}$, and the SAU amplifies the received signal. The data processing unit digitizes the signal at $1 \mathrm{MHz}$, computes cross-correlation between the received and reference signals, and then displays a two-way travel time and quality of the received signal in about $4 \mathrm{~s}$.

Post-cruise processing of the GPS data collected during the experiment were carried out for each of 4-hour segments, with consecutive segments overlapping by 2 hours, using GIPSY/OASIS II software developed at the Jet Propulsion Laboratory (JPL) (Zumberge et al., 1997). We used precise orbits provided by JPL from a global tracking network and interpolate them to each 1-second data point and attempted to fix the phase ambiguities as integer values. We used the JPL satellite clock estimates, nominally given every 5 minutes, as a priori information and estimate each satellite's clock state at 1-second epochs as white noise (Chadwell et al., 1998). We estimate that the horizontal GPS positioning is accurate to 1-2 cm (e.g., Miura et al., 2002; Chadwell et al., 2002).

We calculated a three dimensional position of the underhull acoustic transducer at each transmission and reception of an acoustic signal from the positions of the GPS antennas. 


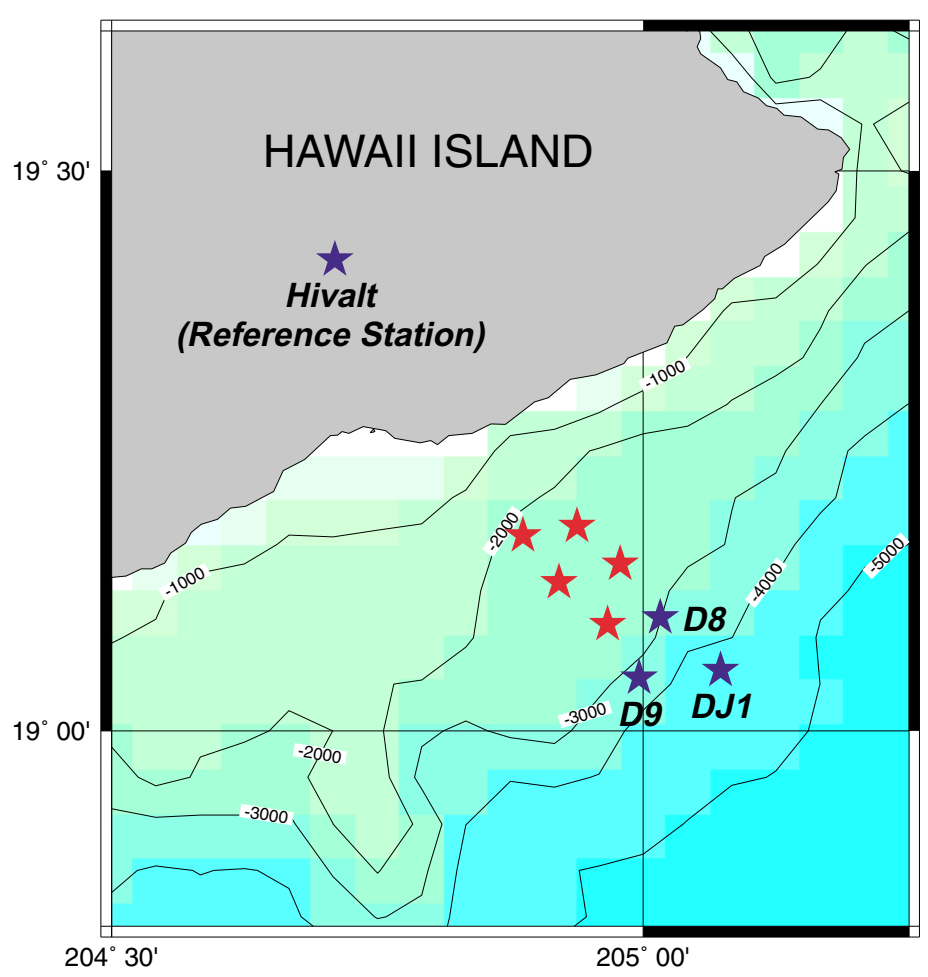

Fig. 1. An array of precision acoustic transponders (PXPs) on the southeastern slope of Hawaii Island deployed in 2000 for monitoring crustal deformation through GPS/A seafloor positioning.

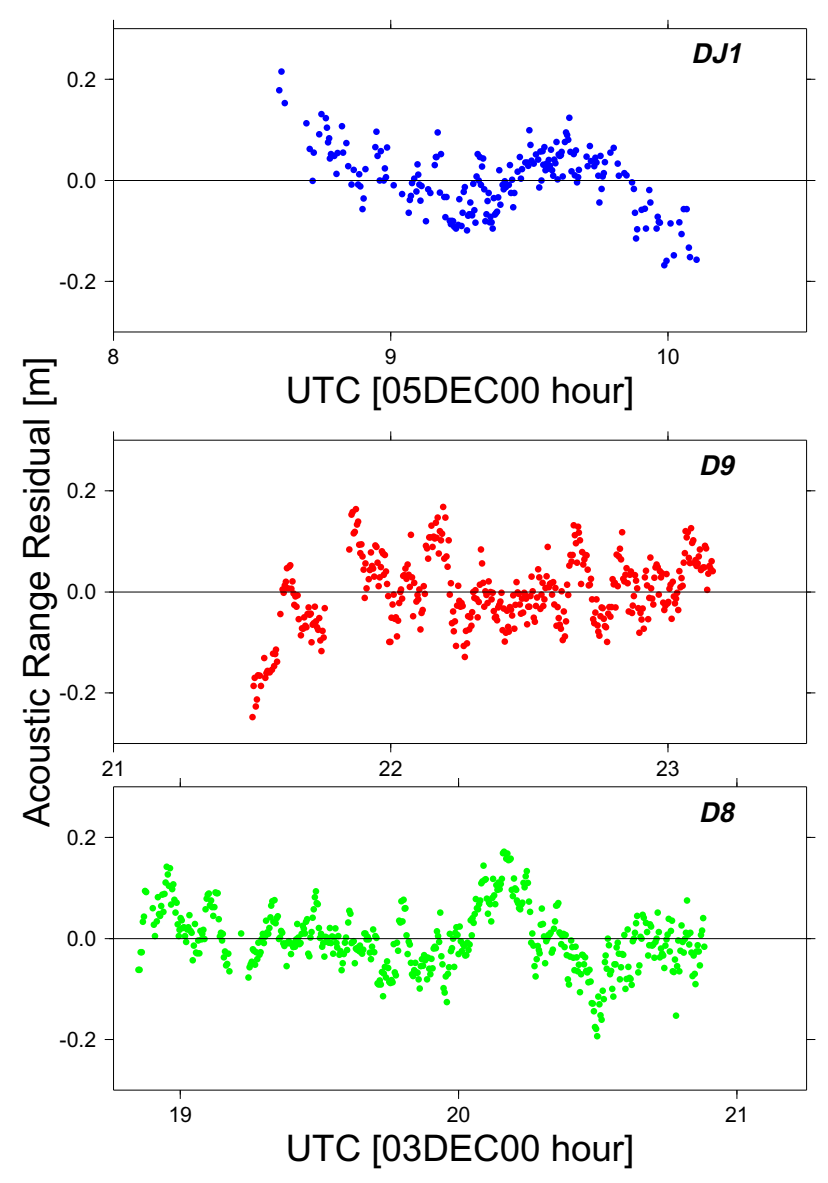

Fig. 2. Acoustic range residuals to each PXP on the bottom during observations around each PXP: D8, D9, DJ1. Velocity structure was assumed to be unchanged.
We assumed the initial position of each PXP with the horizontal position of its deployment and a precise water depth obtained from pressure measurement. Variation of water depths due to ocean tides was observed with GPS positioning. We thus got a geometrical range between the under-hull transducer and the initial position of a PXP on the seafloor.

\section{Correction for the Effect of Sound Velocity Vari- ation}

We need a sound velocity structure to compare the observed acoustic travel time with the geometrical range. Sound velocity in the ocean is a function of temperature, pressure, and salinity or conductivity. The physical structure of the ocean is well approximated with a function of water depth, and it is the surface layer of a few hundreds of meters that is most variable with time. According to the CTD (conductivity, temperature and depth) observations, sound velocity in the upper $500 \mathrm{~m}$ was about $1,507 \mathrm{~m} / \mathrm{s}$ in average, and its variation depended mainly on temperature as was approximated $\Delta C=3.2 \Delta T$, where $\Delta C$ is velocity change $(\mathrm{m} / \mathrm{s})$ and $\Delta T$ is temperature change $\left({ }^{\circ} \mathrm{C}\right)$. If average temperature in the upper $500 \mathrm{~m}$ changes by $1^{\circ} \mathrm{C}$, the effect corresponds to an apparent change of $1.50 \mathrm{~m}$ in acoustic ranging with a ray angle of 45 degrees.

GPS/A seafloor positioning is carried out in two steps assuming a horizontally layered sound velocity structure (Spiess et al., 1998). The first step is GPS/A observations to precisely locate the horizontal position of each PXP. We carried out the observation along a circle around each PXP for about 2 hours with a fixed sound velocity profile obtained from CTD observation. We calculated an acoustic range along a ray path based on the velocity structure and the posi- 


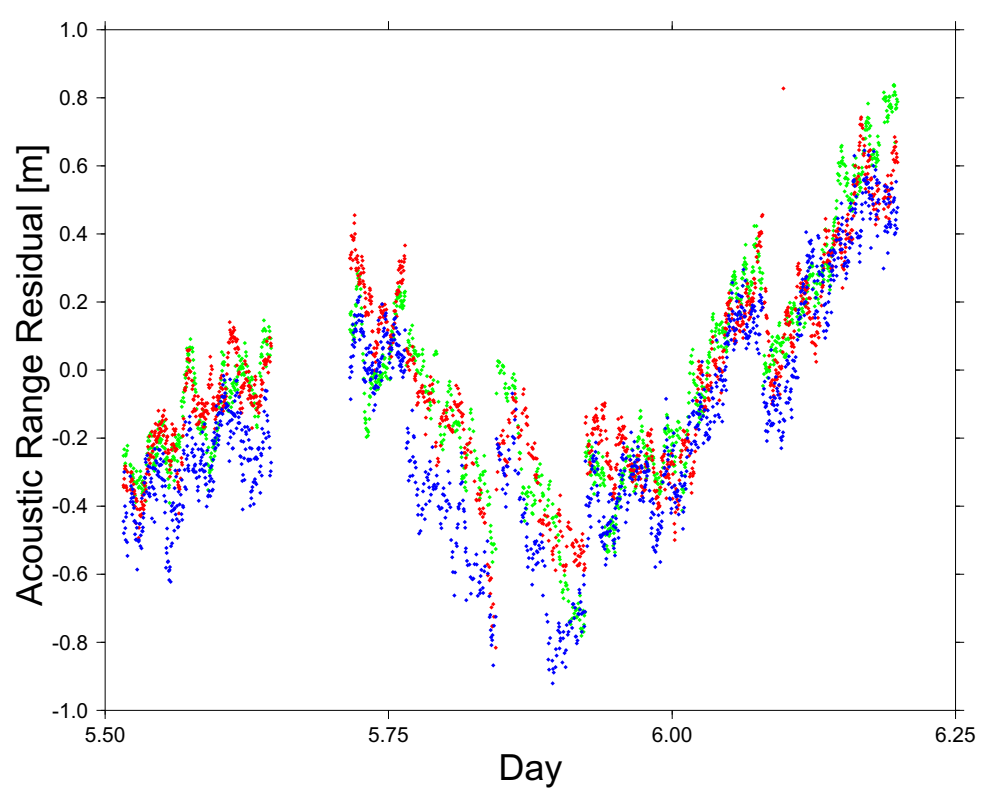

Fig. 3. Acoustic range residuals to each PXP during observations above the array center. The velocity structure was updated with CTD observation about every 3 hours. Colors for the PXPs are the same with Fig. 2. After overlapping mean of 15 minutes, peak-to-peak variations for each PXP are about $+/-0.4 \mathrm{~m}$ except for the last 4 hours without CTD observation.

tions of the under-hull transducer and the PXP. When the ray path is fixed, an observed travel time is converted to an observed acoustic range. Differences between the observed and calculated acoustic ranges are attributed mainly to an error in the initial three-dimensional position of the PXP. The most probable position of each PXP was so estimated as to minimize the differences. Figure 2 shows thus obtained residuals of acoustic range observation.

The second step is GPS/A observations to get a precise horizontal position of the PXP array center. Considering the different water depths of the PXPs, we tried to keep the ship's position near a point where the ray angle to each PXP was the same. Then the ray paths to all the PXPs have the same range within the time-varying surface layer of the ocean. If temporal velocity variations are coherent for all ray paths, they do not shift the array center horizontal position (Spiess et al., 1998).

The residuals of the acoustic range to each PXP amounted to $+/-0.7 \mathrm{~m}$ with a fixed velocity structure. The velocity structure was updated with CTD observation roughly every 3 hours during the observation, and the residuals decreased to $+/-0.4 \mathrm{~m}$ as is shown in Fig. 3 .

It is important that the long-term variations of the residual are correlated among the 3 PXPs as was expected. When the temperature in the surface layer rises, the apparent acoustic ranges to all the PXPs decrease to the same degree, and vice versa. Considering that the range residuals are vectors along the ray paths near the sea surface, we can estimate an effect of velocity variation by calculating a mean of the vertical components of simultaneously observed 3 range residuals. Deviation of the mean from zero shows the effect of sound velocity change. The effect on horizontal components was corrected in this way. Then the position of the underhull transducer obtained from the acoustic positioning should agree to the result from GPS observation, if the positions of the PXPs estimated in the first step are correct. If the re-
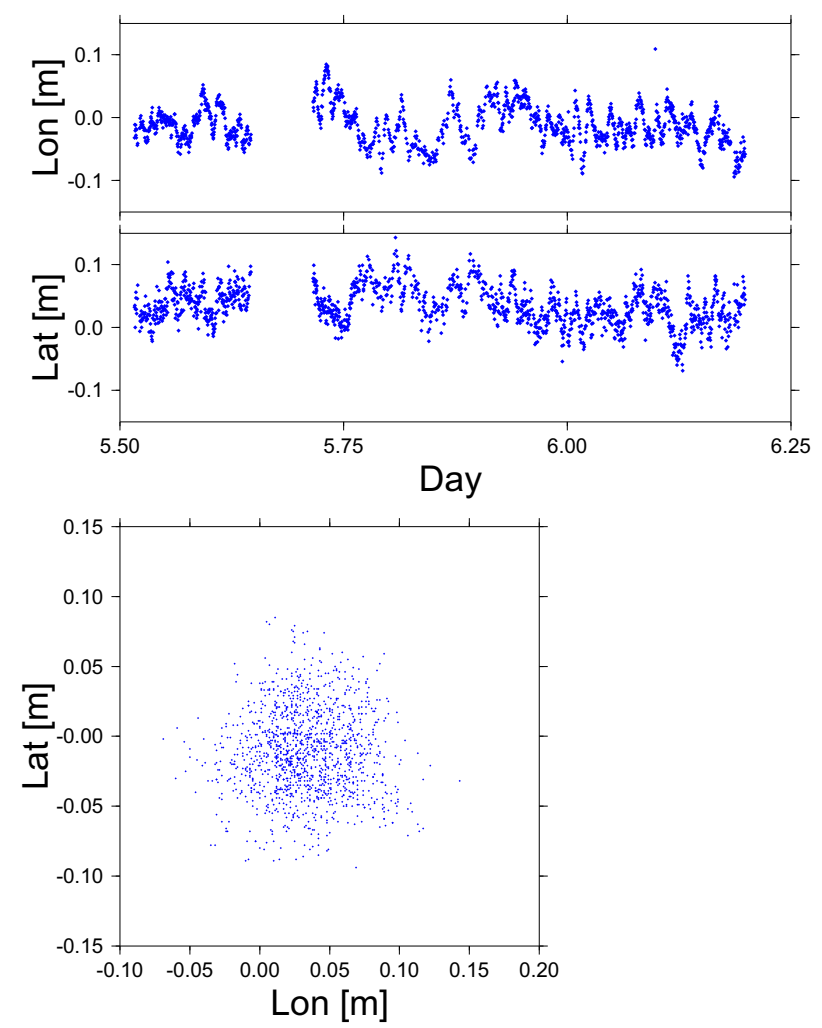

Fig. 4. Differences between the transducer position obtained from GPS observation and that estimated from the acoustic positioning. Effects of the large range residuals shown in Fig. 3 are almost canceled.

sults show a systematic deviation, the simplest interpretation is that there is a bias in the estimated PXPs' positions.

Figure 4 shows the differences between the transducer position obtained from GPS observation and that estimated from the acoustic positioning. Most of the effects of velocity variations shown in Fig. 3 are canceled. The result of posi- 
tioning with RMS residuals is $-1.1+/-3.1 \mathrm{~cm}$ in latitude, and $3.3+/-3.0 \mathrm{~cm}$ in longitude.

\section{Discussions and Summary}

Observed acoustic range residuals could be explained by temperature variations. CTD observation accounted for about one half of the daily variations of the sound velocity structure mainly because the observation every 3 hours could not fully follow the temporal variations. Therefore, observed temperature variation of $+/-0.23^{\circ} \mathrm{C}$ in the upper $500 \mathrm{~m}$ of the ocean accounts for $+/-0.34 \mathrm{~m}$ of the observed acoustic range residuals of $+/-0.7 \mathrm{~m}$. The remaining $+/-0.36 \mathrm{~m}$ corresponds to the results in Fig. 3. Salinity variations were $+/-0.023$ per mil, and the effect was only $+/-0.01 \mathrm{~m}$. Frequent XBT observations would be useful to monitor velocity variations.

Although precision of the positioning in the first step is worse than that of the second step, errors in relative positioning among 3 PXPs have only an indirect effect on estimation of tectonic motion of the seafloor. Furthermore we can improve the accuracy in relative positioning in the future observations.

Considering the points discussed in this paper, GPS/A observation by using 3 PXPs is a promising method for precise seafloor positioning even in the presence of large velocity variations. This method can be applied to seafloor positioning in subduction zones.

Acknowledgments. We thank Dr. J. F. Zumberge and Mr. T. Yabuki for their detailed and constructive review comments. Sup- ported by the Ocean Hemisphere Program of the Ministry of Education, Culture, Sports, Science and Technology, Japan.

\section{References}

Chadwell, C. D., F. N. Spiess, J. A. Hildebrand, L. E. Young, G. Percell, Jr., and H. Dragert, Deep-sea geodesy: Monitoring the ocean floor, GPS World, 9, 44-55, 1998.

Chadwell, C. D., F. N. Spiess, J. A. Hildebrand, and H. Dragert, Seafloor geodetic evidence of episodic spreading $25 \mathrm{~km}$ east of the Juan de Fuca Ridge, EOS Trans. $A G U, \mathbf{8 3}$, Fall Meet. Suppl., Abstract T22A-1130, 2002 .

Fujimoto. H., S. Miura, Y. Osada, H. Shiobara, T. Kanazawa, A. Sweeny, J. A. Hildebrand, and C. D. Chadwell, Deployment of seafloor positioning system for the monitoring of seismic coupling at the Japan Trench off Sanriku, EOS Trans. AGU, 82, Fall Meet. Suppl., Abstract G41A-0212, 2001.

Fujimoto, H., M. Mochizuki, K. Mitsuzawa, T. Tamaki, and T. Sato, Ocean bottom pressure variations in the southeastern Pacific following the 1997-98 El Niño event, Geophys. Res. Lett., 30, 10.1029/ 2002GL016677, 2003.

Miura, S., A. Sweeney, H. Fujimoto, H. Osaki, E. Kawai, R. Ichikawa, T. Kondo, Y. Osada, C. D. Chadwell, Evaluation of accuracy in kinematic GPS analyses using a precision roving antenna platform, EOS Trans. $A G U$, 83, Fall Meet. Suppl., Abstract G52A-0959, 2002.

Spiess, F. N., C. D. Chadwell, J. A. Hildebrand, L. E. Young, G. H. Purcell Jr., and H. Dragert, Precise GPS/Acoustic positioning of seafloor reference points for tectonic studies, Phys. Earth. Planet. Inter., 108, 101-112, 1998.

Zumberge, J. F., M. B. Heflin, D. C. Jefferson, M. M. Watkins, and F. H. Webb, Precise point positioning for the efficient and robust analysis of GPS data from large network, J. Geophys. Res., 102, 5,005-5,017, 1997.

Y. Osada (e-mail: yosada@eri.u-tokyo.ac.jp), H. Fujimoto, S. Miura, A. Sweeney, T. Kanazawa, S. Nakao, S. Sakai, J. A. Hildebrand, and C. D. Chadwell 